\title{
The interaction between protocatechuic aldehyde and human serum albumin using three-dimensional fluorescence techniques
}

\author{
Jing Tian, Changyun Chen* and Mengwei Xue \\ School of Biochemical and Environmental Engineering, Nanjing Xiaozhuang University, Nanjing, \\ China
}

\begin{abstract}
The binding mechanism between protocatechuic aldehyde (PA) and human serum albumin (HSA) was investigated by fluorescence spectroscopy at different temperatures. It is proved that the fluorescence quenching of HSA by PA is not results of the formation of PA-HSA complex. The equilibrium constant $K$ and the number of binding sites $n$ were measured at different temperature by fluorescence quenching method. The standard enthalpy change $(\Delta H)$ and the standard entropy change $(\Delta S)$ of this interaction process were calculated to be $114.02 \mathrm{~kJ} \cdot \mathrm{mol}^{-1}, 541.92 \mathrm{~J} \cdot \mathrm{mol}^{-1} \cdot \mathrm{K}^{-1}$, respectively. According to the interaction between PA and HSA of thermodynamic parameters $\Delta H$ and $\Delta S$, the major acting force can be measured. The synchronous fluorescence spectra shows the microenvironment of tryptophan residue is changed and the hydrophobicity is increased. Through three-dimensional (3D) fluorescence spectra can get some valuable structure changing information.
\end{abstract}

Keywords: Binding mechanism, protocatechuic aldehyde, human serum albumin, three-dimensional (3D) fluorescence spectra

\section{Introduction}

Protocatechuic aldehyde (3,4-dihydroxybenzaldehyde, molecular structure shown in Fig. 1) is one of the most effective component in Salivia miltorrhrza, a Chinese traditional herbal drug, used for treatment of some diseases, such as cardiodynia, myocardial infarction, phylogenic infection, hypochondriac pain, stomachache dysmenorrhea and trauma with blood stasis. This molecule can treat anti-cardium colic, improve flow in coronary arteries and inhibit the aggregation of platelets caused by ADP (adenosine diphosphate) [6]. It also can be used as a precursor in the vanillin synthesis by biotransformation by Capsicum frutescens cell cultures [7].

Human serum albumin (HSA), as the most abundant protein constituent of blood plasma, has a high affinity to an extraordinarily diverse range of materials, such as drugs, metabolites, fatty acids and metal ions [1]. As a kind of storage form of chemicals, it plays an important role on the disposition and transportation of drugs and other exogenous and endogenous compounds to their targets. It serves as a solubilizer and transporter for the materials and it is also responsible for the maintenance of blood $\mathrm{pH}$, the drug disposition and efficacy, and the contribution of colloid osmotic blood pressure [4,5,8].

In this paper, the interaction between protocatechuic aldehyde (PA) and HSA has been investigated with fluorescent spectrum under physiological conditions.

\footnotetext{
${ }^{*}$ Corresponding author: Changyun Chen, School of Biochemical \& Environmental Engineering, Nanjing Xiaozhuang University, Nanjing 211171, China. E-mail: yhjiangccy@126.com.
} 
<smiles>O=Cc1ccc(O)c(O)c1</smiles>

Fig. 1. Molecular structure of protocatechuic aldehyde.

\section{Materials and methods}

\subsection{Instruments and materials}

The fluorescence spectra were recorded on a Perkin Elmer LS-50B fluorophotometer (USA).

Human serum albumin (HSA) was purchased from Sigma Chemical Company. It was used without further purification and its molecular weight was assumed to be $66,500 \mathrm{HSA}(0.5 \mu \mathrm{M})$ solutions were prepared in (pH 7.4). Protocatechuic aldehyde was provided by China Pharmaceutical University, with a concentration of $54.8 \mu \mathrm{g} / \mathrm{ml}$. All other reagents and solvents were of analytical reagent grade.

\subsection{Methods}

Fluorescence spectra of HSA ( $3 \mathrm{ml} 0.5 \mu \mathrm{M}$ in a $1.0 \mathrm{~cm}$ quartz cell) were recorded in the absence and presence of different amount of trace drug solutions.

The $\mathrm{pH}$ measurements were carried out on a pHS-25 Exact Digital pH meter.

\section{Results and discussion}

\subsection{Fluorescence spectra}

\subsubsection{Fluorescence emission spectra}

Figure 2 shows the emission spectra of HSA in the absence and presence of PA. As can be seen from Fig. 2, HSA has a strong fluorescence emission with a peak at $350 \mathrm{~nm}$ upon excitation at $280 \mathrm{~nm}$, and its fluorescence intensity decreases in the presence of PA. It implies that the binding of PA to HSA occurs and the microenvironment around the chromophore of HSA is changed. There is about $25 \mathrm{~nm}$ wavelength redshift on line $\mathrm{h}$. Shift of maximum emission $\left(\lambda_{\max }\right)$ is an indication that the conformation of protein can be affected by the addition of the drug. The results indicate that there are strong interactions between PA and HSA.

\subsubsection{The relations between temperatures and fluorescence quenching}

In order to confirm the quenching mechanism, the fluorescence quenching was analyzed according to the well-known Stern-Volmer equation:

$$
F_{0} / F=1+K_{\mathrm{q}} \tau_{0}[Q]=1+K_{\mathrm{SV}}[Q]
$$

where $F_{0}$ and $F$ are the fluorescence intensities in the absence and presence of PA, respectively. [Q] is the concentration of PA and $K_{\mathrm{SV}}$ is the Stern-Volmer quenching constant. $K_{\mathrm{q}}$ is the quenching rate constant of the biomolecule, and $\tau_{0}$ is the average lifetime of the biomolecule without quencher. 


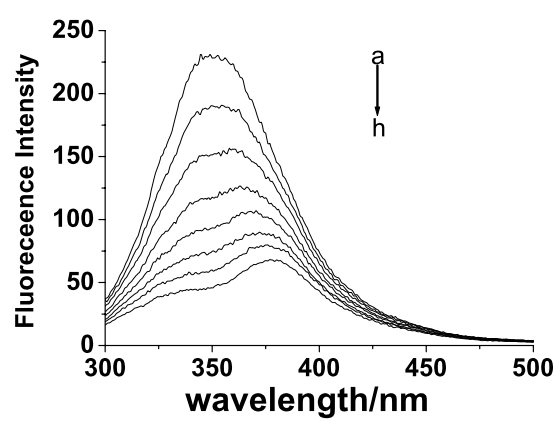

Fig. 2. Fluorescence emission spectra (a) $5.0 \times 10^{-7} \mathrm{~mol} / \mathrm{l} \mathrm{HSA}$, (b)-(h) $5.0 \times 10^{-7} \mathrm{~mol} / \mathrm{l}$ HAS in the presence of $3.97 \times 10^{-7} \mathrm{~mol} / 1,7.94 \times 10^{-7} \mathrm{~mol} / 1,11.91 \times 10^{-7} \mathrm{~mol} / 1,15.88 \times 10^{-7} \mathrm{~mol} / 1,19.85 \times 10^{-7} \mathrm{~mol} / 1,23.82 \times 10^{-7} \mathrm{~mol} / 1$, $27.79 \times 10^{-7} \mathrm{~mol} / \mathrm{l} \mathrm{PA}$, respectively.

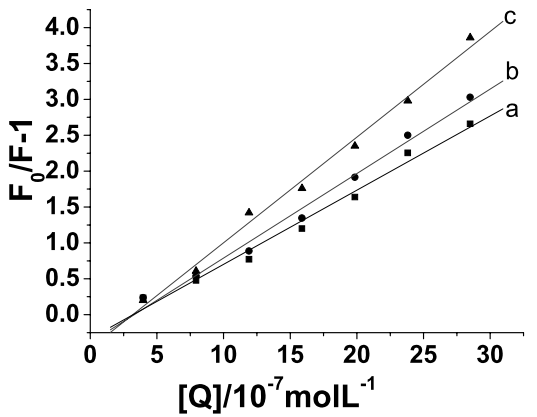

Fig. 3. The Stern-Volmer curve of HSA $\left(5.0 \times 10^{-7} \mathrm{~mol} / \mathrm{l}^{-1}\right)$ quenched by PA (a) $288 \mathrm{~K}$; (b) $298 \mathrm{~K}$; (c) $308 \mathrm{~K}$.

In order to illustrate the fluorescence quenching mechanism of HSA by PA, the fluorescence intensity data was analyzed according to the Stern-Volmer equation by plotting $F_{0} / F$ versus the PA concentration $[Q]$ at 288,298 and $308 \mathrm{~K}$, respectively (see Fig. 3). It can be obviously observed that $K_{\mathrm{SV}}$ increases while the temperature increasing, therefore, the fluorescence quenching mechanism is dynamic quenching, which resulting from collisional encounters between the fluorophore and quencher. It is also shown that the binding between PA and HSA is remarkable and the effect of temperature is obvious.

\subsection{Synchronous fluorescence}

The method of synchronous fluorescence is a kind of simple and effective way to measure the fluorescence quenching and the possible shift of the maximum emission wavelength. The fluorescence of HSA comes from tyrosine, tryptophan and phenylalanine residues. According to Miller [3], with large $\Delta \lambda$ values such as $60 \mathrm{~nm}$, the synchronous fluorescence of HSA is characteristic of tryptophan residue. Figure 4 shows the effect of addition of PA on the synchronous fluorescence spectra of HSA when $\Delta \lambda=60 \mathrm{~nm}$. It is indicated that addition of PA results in the strong fluorescence quenching of tryptophan residues and significant blue shift is observed when the density of PA increased from 0 to $27.97 \times 10^{-7} \mathrm{~mol} / \mathrm{l}$. This shows the microenvironment of tryptophan residue is changed and the hydrophobicity is increased. 


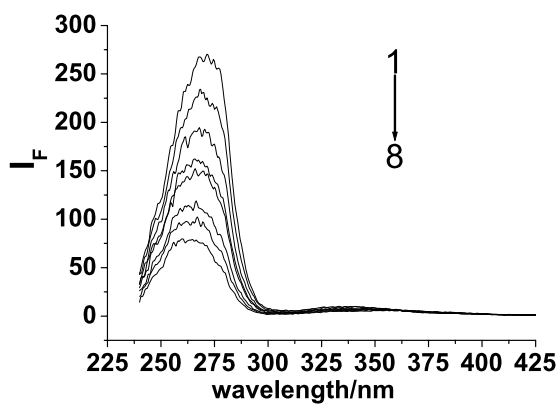

Fig. 4. Synchronous fluorescence spectra of HSA $\left(5.0 \times 10^{-7} \mathrm{~mol} / \mathrm{l}\right)$ with $\Delta \lambda=60 \mathrm{~nm}$ in the absence and presence of increasing amount of PA from 1 to 8: 0, 0.397, 0.794, 1.191, 1.588, 1.985, 2.382, $2.779 \mu \mathrm{mol} / \mathrm{l}$.

Table 1

The binding sites number $n$ and binding constant $K$ of PA-HSA at different temperatures

\begin{tabular}{lcc}
\hline$T(\mathrm{~K})$ & $n$ & $K\left(\times 10^{7} \mathrm{M}^{-1}\right)$ \\
\hline 288 & 1.10 & 4.43 \\
298 & 1.17 & 13.3 \\
308 & 1.22 & 97.9 \\
\hline
\end{tabular}

\subsection{Determinations of binding sites number and binding constant}

From the equation: $\lg \left[\left(F_{0}-F\right) / F\right]=\lg K+n \lg [Q]$ [2], binding sites number $n$ can be obtained and listed in Table 1 together with binding constant $K$. It can be observed that the average binding sites number $n$ is one, and binding constant $K$ increased while the temperature increased.

\subsection{Determination of the acting force}

Essentially, there are four types of non-covalent interaction existing in the binding of the ligands to proteins. These are hydrogen bonds, van der Waals forces, hydrophobic and electrostatic interactions [3]. Thermodynamic parameters, free energy $(\Delta G)$, standard enthalpy $(\Delta H)$ and standard entropy $(\Delta S)$ can provide an insight into the binding mode. Among these parameters, $\Delta G$ reflects the possibly of reaction, $\Delta H$ and $\Delta S$ are the main evidence to determine acting forces. When temperature varies in a small range, the $\Delta H$ can be considered as a constant.

Therefore, from the following equations:

$$
\begin{aligned}
& \ln \left(K_{2} / K_{1}\right)=\Delta H\left(1 / T_{1}-1 / T_{2}\right) / R, \\
& \Delta G=\Delta H-T \Delta S=-T \ln K .
\end{aligned}
$$

These thermodynamics parameters are calculated from the linear relationship between $\ln K$ and the $1 / T$ (Table 2). $\Delta H$ and $\Delta S$ of the binding interaction between PA and HSA are calculated to be $114.02 \mathrm{~kJ} \cdot \mathrm{mol}^{-1}$ and $541.92 \mathrm{~J} \cdot \mathrm{mol}^{-1} \cdot \mathrm{K}^{-1}$. Because enthalpy change $\Delta H>0$ and entropy change $\Delta S>0$, the acting force, which result in the form of the non-fluorescence complex, are mainly caused by electrostatic and hydrophobic interaction [3,5]. 
Table 2

Thermodynamic parameters of the system of PA-HAS

\begin{tabular}{lccccc}
\hline $\begin{array}{l}\text { Temperature } \\
(\mathrm{K})\end{array}$ & $\begin{array}{c}K \\
\left(\times 10^{7} \mathrm{l} \cdot \mathrm{mol}^{-1}\right)\end{array}$ & $\begin{array}{c}\Delta G^{\Theta} \\
\left(\mathrm{kJ} \cdot \mathrm{mol}^{-1}\right)\end{array}$ & $R$ & $\begin{array}{c}\Delta S^{\Theta} \\
\left(\mathrm{J} \cdot \mathrm{mol}^{-1} \mathrm{~K}^{-1}\right)\end{array}$ & $\begin{array}{c}\Delta H^{\Theta} \\
\left(\mathrm{kJ} \cdot \mathrm{mol}^{-1}\right)\end{array}$ \\
\hline 288 & 4.43 & -42.16 & 0.9974 & 541.92 & 114.02 \\
298 & 13.3 & -47.25 & & & \\
308 & 97.9 & -53.01 & & & \\
\hline
\end{tabular}

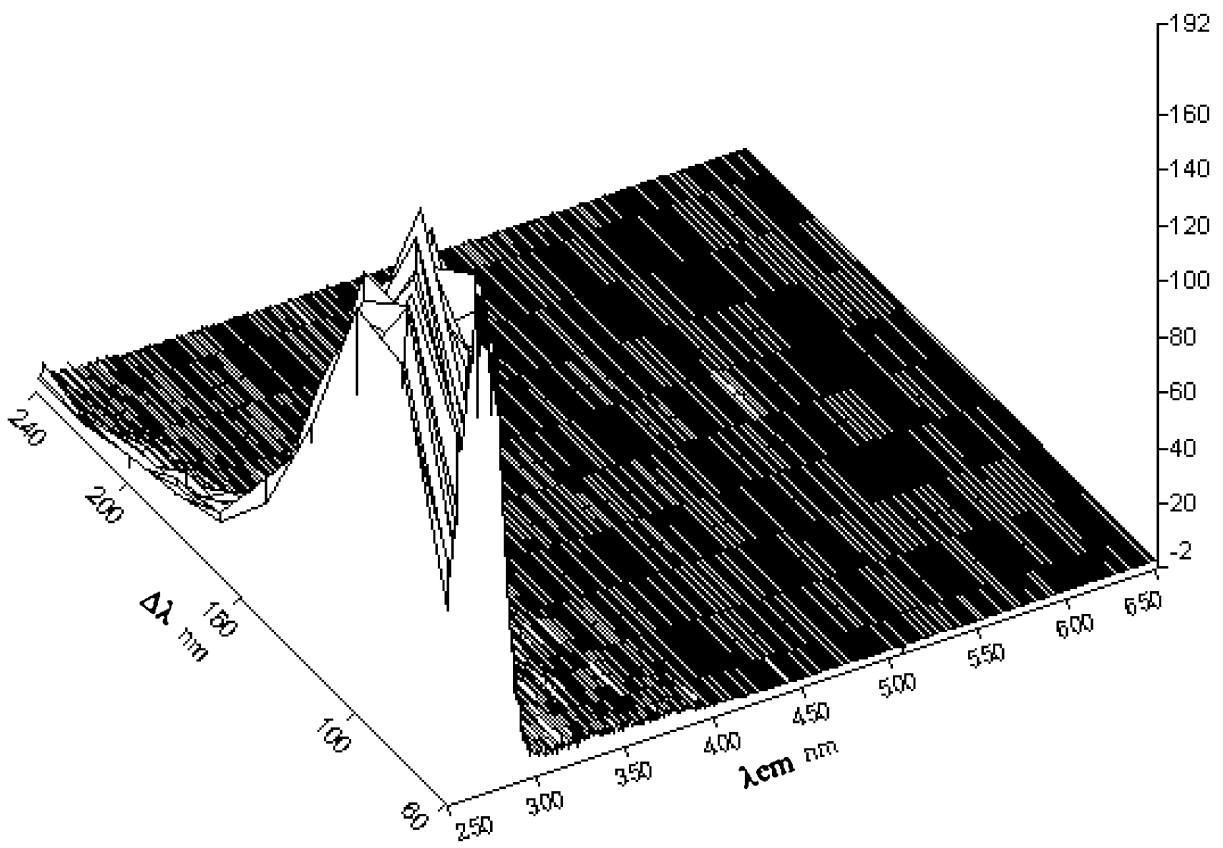

Fig. 5. The three-dimensional projections of $2 \mathrm{ml} \mathrm{HSA}\left(C_{\mathrm{HSA}}: 5.0 \times 10^{-7} \mathrm{~mol} / \mathrm{l}, C_{\mathrm{PA}}: 3.97 \times 10^{-4} \mathrm{~mol} / \mathrm{l}\right)$.

\subsection{The three-dimensional fluorescence spectra}

The three-dimensional fluorescence spectra are a promising fluorescence analysis technique in recent years. Figures 5-7 presented the three-dimensional fluorescence spectrum and their characteristics of PA-HSA, respectively. It was apparent that the shift in position of $D$-value $(\Delta \lambda)$ (between excitation wavelength and emission wavelength) were ranged from about 150-175 $\mathrm{nm}$. The result showed that the emission maximum of tryptophan residues did significant blue shift while tyrosine residues did red shift. The polarity around some of the tyrosine residues decreased while tryptophan residues increased. This may be due to the changes of residue microenvironment with the insertion of PA.

\section{Conclusions}

This paper indicated that PA can quench the intrinsic fluorescence of HSA, and the quenching mechanisms is dynamic quenching. In addition, the standard enthalpy change $(\Delta H)$ and the standard entropy change $(\Delta S)$ of this interaction process were calculated to be $114.02 \mathrm{~kJ} \cdot \mathrm{mol}^{-1}$ and $541.92 \mathrm{~J} \cdot \mathrm{mol}^{-1} \cdot \mathrm{K}^{-1}$, which indicated that hydrophobic and electrostatic forces played major roles in the interaction between 


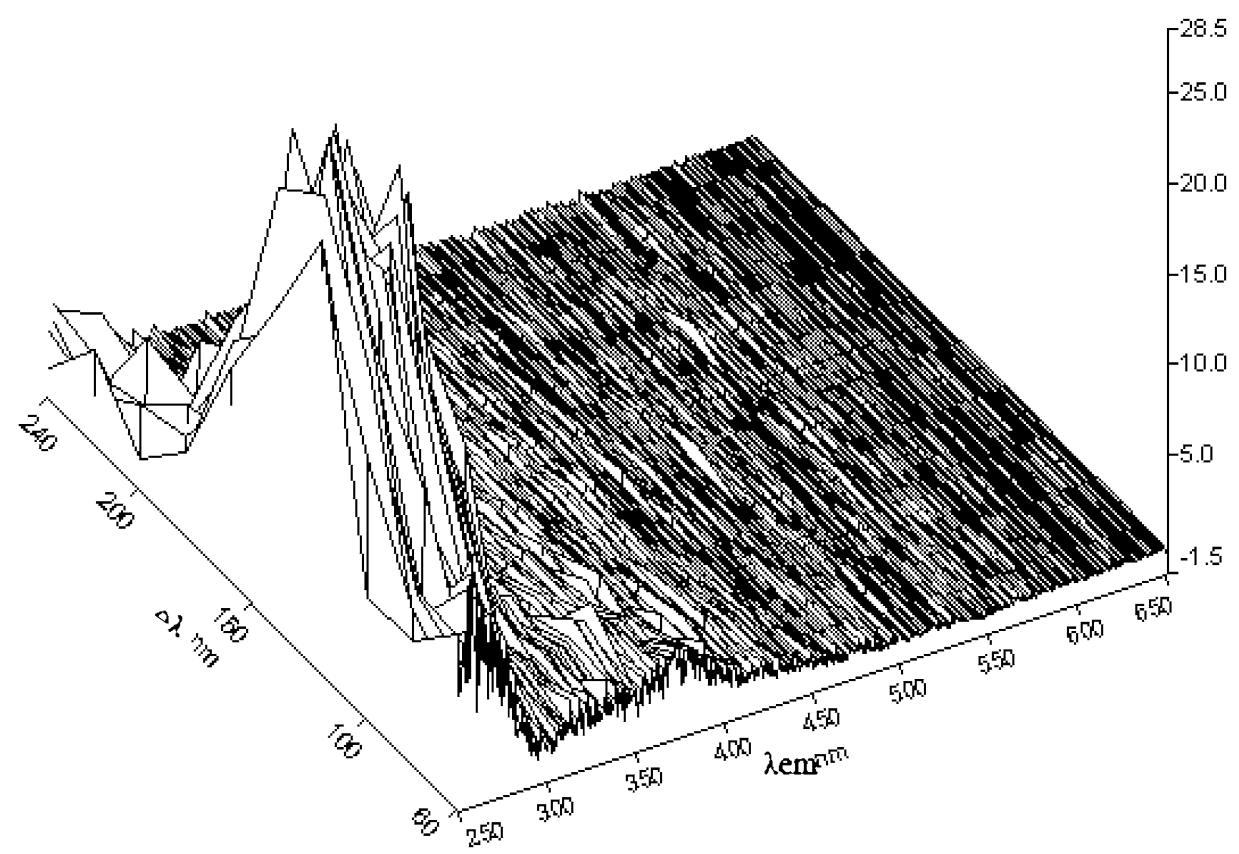

Fig. 6. The three-dimensional projections of $2 \mathrm{ml} \mathrm{HSA}-0.5 \mathrm{ml} \mathrm{PA}\left(C_{\mathrm{HSA}}: 5.0 \times 10^{-7} \mathrm{~mol} / 1, C_{\mathrm{PA}}: 3.97 \times 10^{-4} \mathrm{~mol} / \mathrm{l}\right)$.

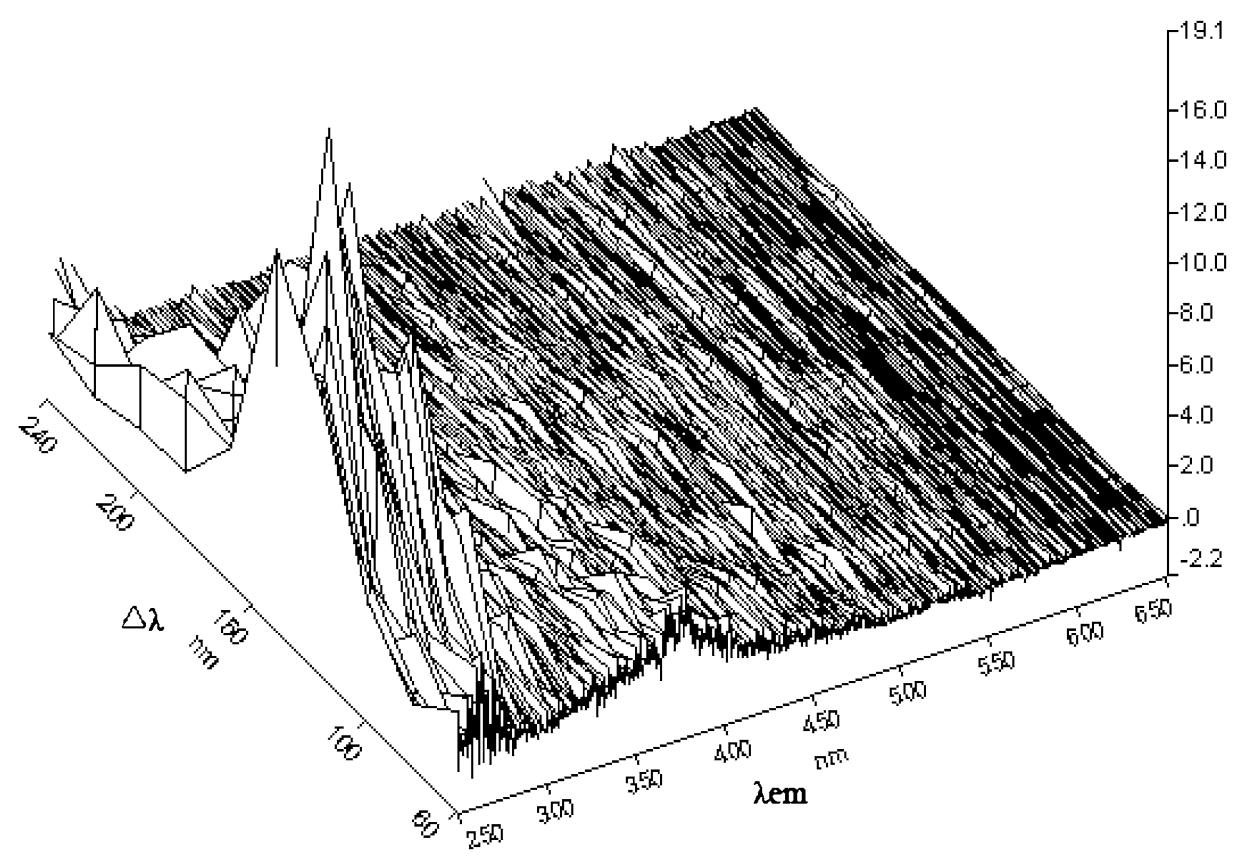

Fig. 7. The three-dimensional projections of $2 \mathrm{ml} \mathrm{HSA}-1 \mathrm{ml} \mathrm{PA}\left(C_{\mathrm{HSA}}: 5.0 \times 10^{-7} \mathrm{~mol} / \mathrm{l}, C_{\mathrm{PA}}: 3.97 \times 10^{-4} \mathrm{~mol} / \mathrm{l}\right)$.

PA and HSA. And the result of 3D fluorescence spectra indicated that the polarity around the tyrosine residues decreased while tryptophan residues increased, resulted in a disordered structure of HSA. 


\section{References}

[1] D.C. Carter and J.X. Ho, Advances in Protein Chemistry, Academic Press, San Diego, CA, 1994.

[2] C.Y. Chen, K. Chen, Q. Long, M.H. Ma and F. Ding, Structural characterization and DNA-binding properties of Sm(III) complex with ofloxacin using spectroscopic methods, Spectroscopy 23 (2009), 103-111.

[3] C.Y. Chen, M.H. Ma, J.Q. Zhang, L.C. Wang and B.R. Xiang, Spectroscopic investigation of the interaction of bovine serum albumin with a novel cardiac agent V-09, Spectroscopy 22 (2008), 43-50.

[4] C.Y. Chen, B.R. Xiang, L.Y. Yu, T. Wang and B. Zhao, The application of two-dimensional fluorescence correlation spectroscopy on the interaction between bovine serum albumin and paeonolum in the presence of Fe(III), Spectrosc. Lett. 41 (2008), 385-392.

[5] C.Y. Chen, J.H. Zhou and X.T. Gu, Binding studies of paeonolum with bovine serum albumin using spectroscopic methods, Spectroscopy 21 (2007), 53-60.

[6] Y. Pan, L. Zhang and G. Chen, Separation and determination of protocatechuic aldehyde and protocatechuic acid in Salivia miltorrhrza by capillary electrophoresis with amperometric detection, Analyst 126 (2001), 1519-1523.

[7] S.R. Rao and G.A. Ravishankar, Biotransformation of protocatechuic aldehyde and caffeic acid to vanillin and capsaicin in freely suspended and immobilized cell cultures of Capsicum frutescens, J. Biotechnol. 76(2,3) (2000), 137-146.

[8] K. Yamasaki, T. Maruyama, U. Kragh-Hansen and M. Otagiri, Characterization of site I on human serum albumin: concept about the structure of a drug binding site, Biochem. Biophys. Acta 1295 (1996), 57-147. 


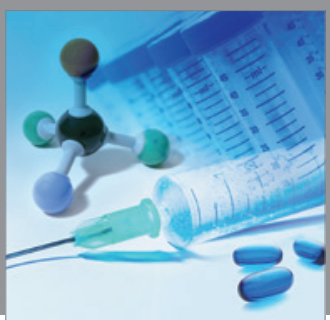

International Journal of

Medicinal Chemistry

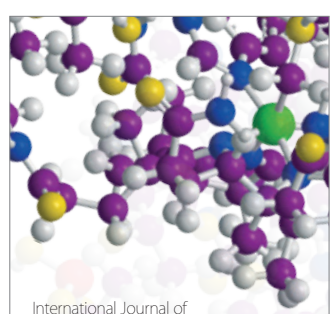

Carbohydrate Chemistry

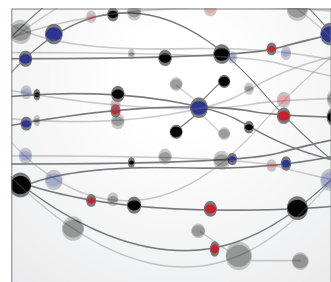

The Scientific World Journal
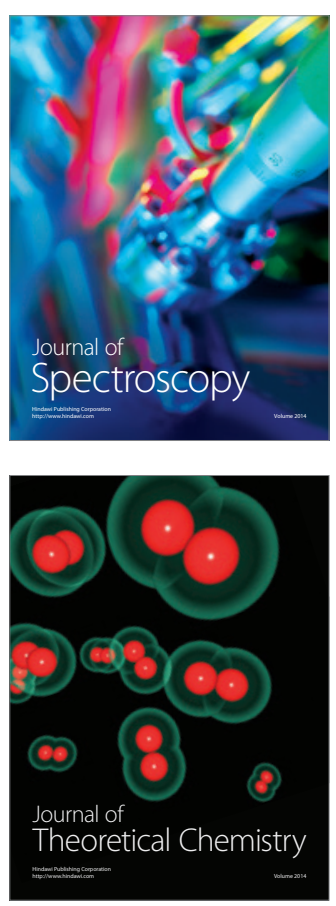
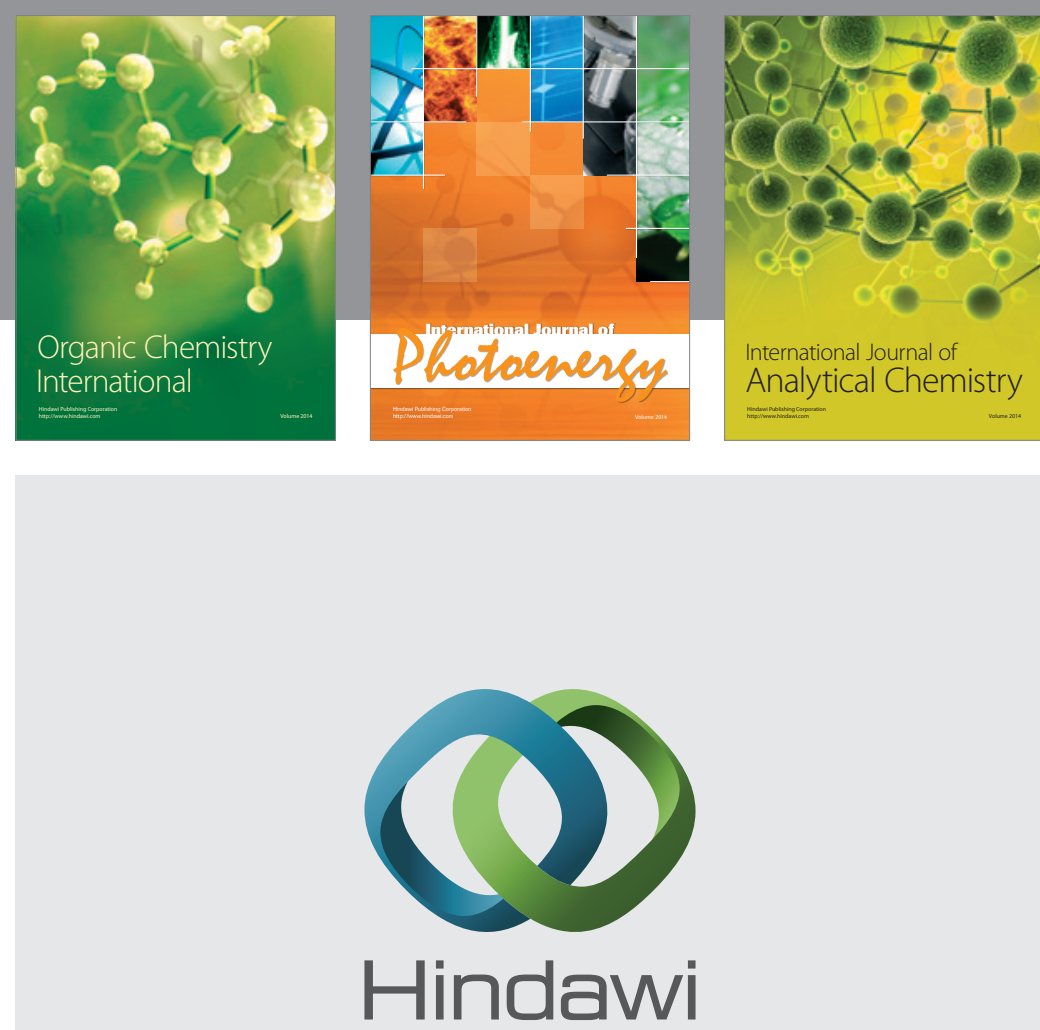

Submit your manuscripts at

http://www.hindawi.com
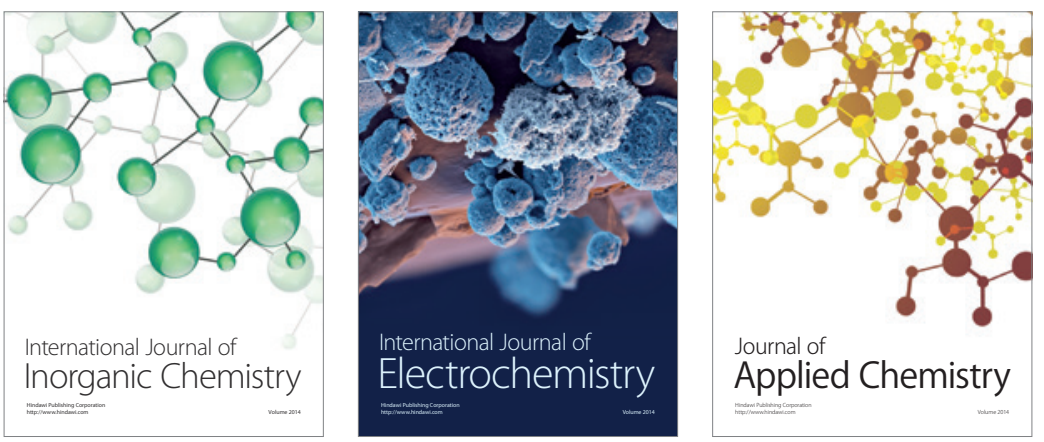

Journal of

Applied Chemistry
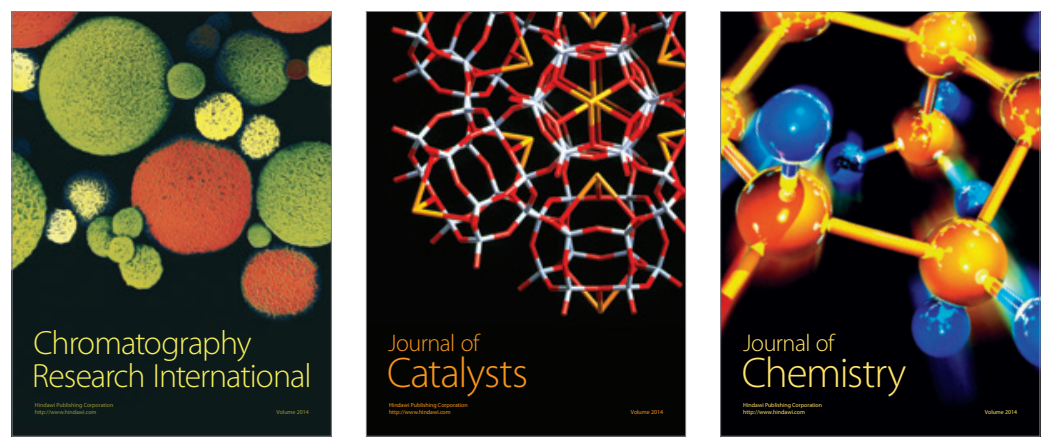
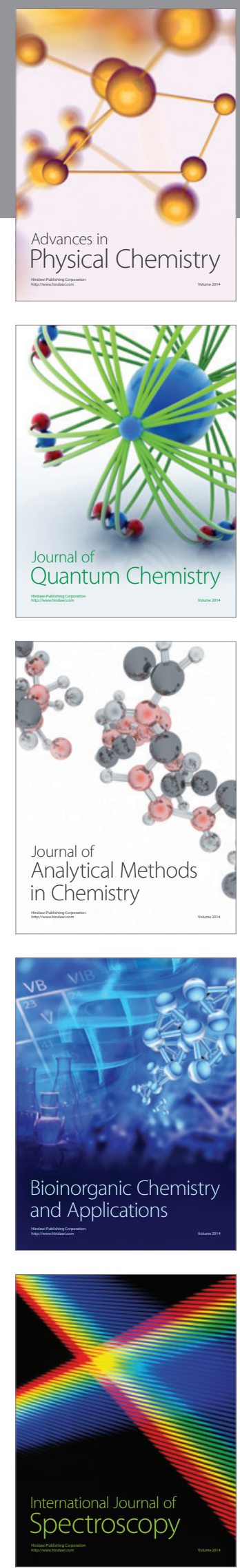